\title{
Hot electron effects in unipolar n-type submicron structures based on GaN, AIN and their ternary alloys
}

\author{
C. Sevik and C. Bulutay
}

\begin{abstract}
The authors present an analysis of impact ionisation (II) and related hot electron effects in submicron sized GaN, AlN and their ternary alloys, all of which can support very high field regimes, reaching a few megavolts per centimetre $(\mathrm{MV} / \mathrm{cm})$. The proposed high field transport methodology is based on the ensemble Monte Carlo technique, with all major scattering mechanisms incorporated. As a test-bed for understanding II and hot electron effects, an $n^{+}-n-n^{+}$channel device is employed having a $0.1 \mu$ m thick $n$-region. The time evolution of the electron density along the device is seen to display oscillations in the unintentionally doped $\mathrm{n}$-region, until steady state is established. The fermionic degeneracy effects are observed to be operational especially at high fields within the anode $\mathrm{n}^{+}$-region. For $\mathrm{Al}_{x} \mathrm{Ga}_{1-x} \mathrm{~N}$-based systems, it can be noted that due to alloy scattering, carriers cannot acquire the velocities attained by the GaN and AlN counterparts. Finally, at very high fields II is shown to introduce a substantial energy loss mechanism for the energetic carriers that have just traversed the unintentionally doped n-region.
\end{abstract}

\section{Introduction}

GaN, AlN and their ternary alloys are becoming technologically important semiconductors, finding application in high-power microelectronic devices such as GaN/AlGaN HEMTs as well as in optoelectronic devices such as visible- and solar-blind AlGaN photodiodes. Impact ionisation (II) is an important process for all these devices subject to extreme electric fields. In the case of highpower devices, II is undesired, leading to breakdown, whereas the operation of devices such as avalanche photodiodes relies on the II mechanism. The subject of this work is the analysis of II and related hot electron effects in GaN, AlN and their ternary alloys, all of which can support very high field regimes, reaching values of a few $\mathrm{MV} / \mathrm{cm}$.

Surprisingly, there has been, as yet, no published measurement of the II coefficient for the $\mathrm{Al}_{x} \mathrm{Ga}_{1-x} \mathrm{~N}$ system. To meet this demand from the computational side, very recently we analysed II in bulk AlGaN alloys [1], whereas in this work, we focus on device-related aspects of II and hot electron effects. A useful model system for understanding hot electron effects is the unipolar $\mathrm{n}^{+}-\mathrm{n}-\mathrm{n}^{+}$homojunction channel, which is to some extent impractical as it gives rise to excessive current density. In the following Sections we explain our computational

\section{(C) IEE, 2003}

IEE Proceedings online no. 20030047

DOI: 10.1049/ip-opt:20030047

Paper received 11 th November 2002

The authors are with the Department of Physics, Bilkent University, Bilkent 06533, Ankara, Turkey procedure and present the transient and steady-state hot electron phenomena taking place within this structure.

\section{Computational approach}

The proposed high-field transport methodology is based on the ensemble Monte Carlo technique [2], incorporating standard scattering processes: acoustic, non-polar and polar optical phonons, ionised impurity scattering, as well as alloy and II scatterings. We include all of the valleys in the lowest two conduction bands, aided by empirical pseudopotential band structure calculations $[3,4]$. We further append an additional higher-lying free electron band to account for the remaining bands. Because the emphasis is on II, the energy loss of the impacting electrons is accurately modelled [1]. We employ more than 20000 electrons within the ensemble, for a total simulation time of about $7.5 \mathrm{ps}$. The time interval to invoke the Poisson solver is taken to be $0.1 \mathrm{fs}$.

The subject of alloy scattering has caused much controversy over the years, which is still unsettled. In the case of group-III nitrides, Farahmand et al. [5] dealt with this issue and reported that using the conduction band offset between the binary constituents as the alloy potential leads to an upper bound for alloy scattering. Being more conservative, for this value we prefer to use $0.91 \mathrm{eV}$, which is half of the corresponding GaN/AIN conduction band offset. Another source of concern has to do with the particular implementation of alloy scattering within the Monte Carlo simulation. Following Fischetti and Laux [6], we treat the alloy scattering as an intra-valley process with the distribution of the final scattering angles assumed to be isotropic, even though at higher energies it attains a forward directional 
character, which should presumably, weaken the effect of this mechanism on momentum relaxation. Therefore, we are led to think that the effect of alloy scattering may still be overestimated.

\section{Results}

To highlight the high-field transport phenomena in GaN, AlN or $\mathrm{Al}_{x} \mathrm{Ga}_{1-x} \mathrm{~N}$ based structures with submicron sized dimensions we consider a simple $\mathrm{n}^{+}-\mathrm{n}-\mathrm{n}^{+}$homojunction channel device [7] having $0.1 \mu \mathrm{m}$ thick unintentionally doped $\left(10^{16} \mathrm{~cm}^{-3}\right)$ n-region sandwiched between two heavily doped $\left(5 \times 10^{18} \mathrm{~cm}^{-3}\right) \mathrm{n}^{+}$-regions of thickness at least $0.2 \mu \mathrm{m}$. Fig. 1 shows the velocity profiles for these materials; the $\mathrm{Al}_{0.4} \mathrm{Ga}_{0.6} \mathrm{~N}$-based structure suffers severely from alloy scattering and has a much reduced velocity. If we turn off the alloy scattering, then the curve for $\mathrm{Al}_{0.4} \mathrm{Ga}_{0.6} \mathrm{~N}$ (not shown) almost coincides with that for $\mathrm{GaN}$. Previous analysis of bulk AlGaN alloys has shown that alloy scattering can modify the high-energy electron distribution and lead to an increased II threshold [1].

The electric field along this device is distributed highly non-uniformly, reaching a values of a few $\mathrm{MV} / \mathrm{cm}$, which peaks at the right $\mathrm{nn}^{+}$interface, as shown in Fig. 2. Also note the penetration of the electric field into the heavily doped anode $\mathrm{n}^{+}$-region with increasing applied bias, which amounts to widening of the unintentionally n-doped 'base' region, as in the Kirk effect. The time evolution of the electron density profile is depicted in Fig. 3 starting at $0.4 \mathrm{ps}$. Oscillations around the unintentionally doped n-region are clearly visible until steady state is established ( 7.5 ps curve in Fig. 3). Fermionic degeneracy effects are seen to be operational at high fields and at high concentration spots. We make use of the Lugli-Ferry recipe [8] to account for degeneracy. However, if degeneracy is ignored, the electron distribution is observed to develop a dip in the $\mathrm{n}^{+}$-anode region, shown in Fig. 4. At a higher applied bias ( $80 \mathrm{~V}$ in $\mathrm{GaN}$ ) the effect of $\mathrm{II}$ becomes dominant. As illustrated in Fig. 5, this mechanism introduces a substantial energy loss mechanism for those energetic carriers that have just traversed the unintentionally doped $n$-region.

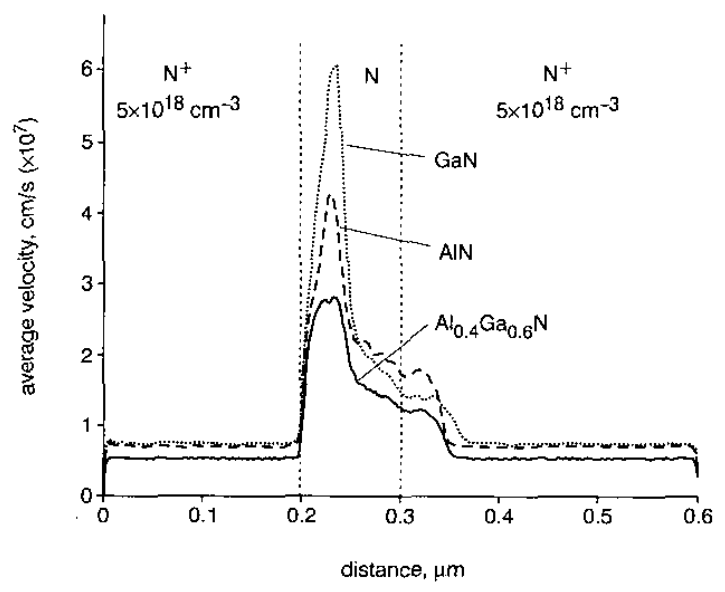

Fig. 1 Velocity distribution over $n^{+}-n-n^{+}$channel under applied bias of $20 \mathrm{~V}$

IEE Proc.-Optoelectron., Vol. 150, No. 1, February 2003

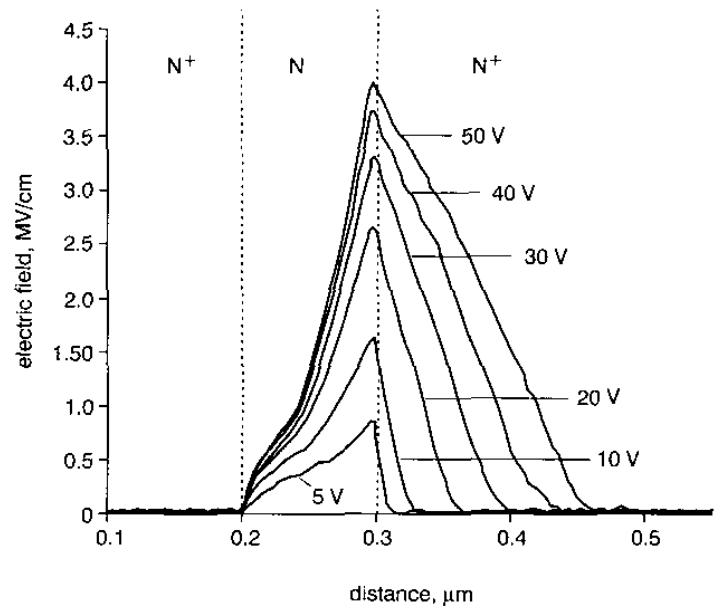

Fig. 2 Electric field distribution over $n^{+}-n-n^{+}$GaN channel at applied biases ranging from $5 \mathrm{~V}$ to $50 \mathrm{~V}$

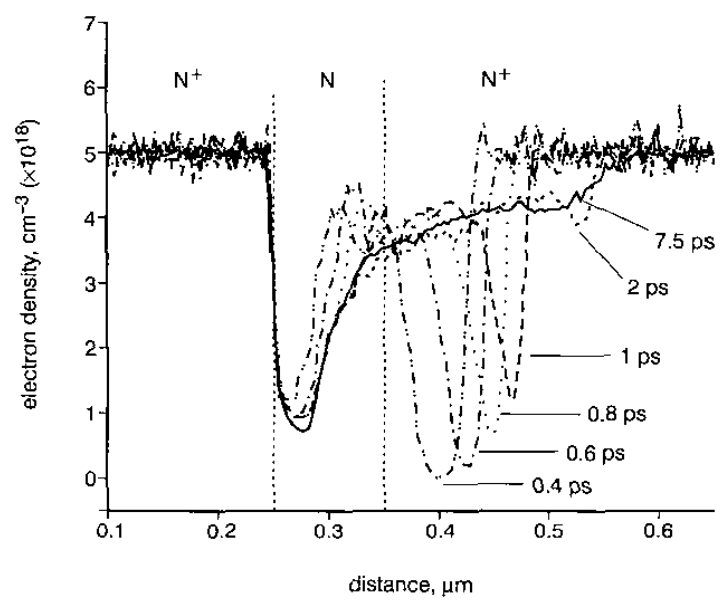

Fig. 3 Time evolution of transient electron density profile over $n^{+}-n-n^{+}$GaN channel under applied bias of $50 \mathrm{~V}$

Stcady-state result is also shown, evaluated at $7.5 \mathrm{ps}$

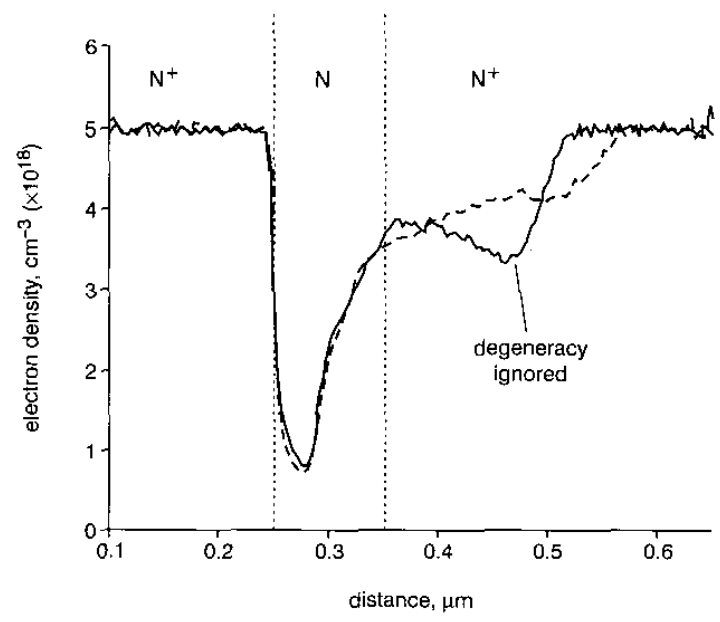

Fig. 4 Steady-state density profile at bias of $50 \mathrm{~V}$, with and without degeneracy effects included 


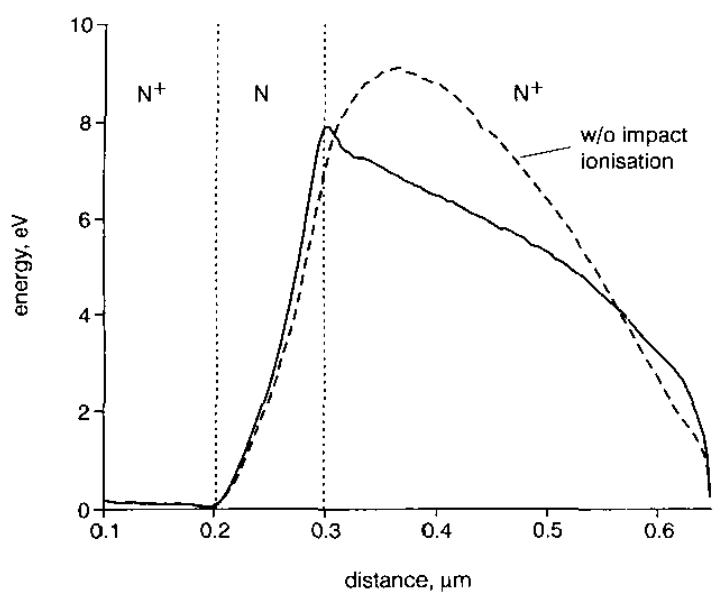

Fig. 5 Energy distribution over $n^{+}-n-n^{+}$GaN channel under applied bias of $80 \mathrm{~V}$, with and without impact ionisation included

\section{Summary}

We have studied high-field transport in submicron sized $\mathrm{n}$-doped unipolar structures. Alloy scattering in the case of $\mathrm{Al}_{x} \mathrm{Ga}_{1-x} \mathrm{~N}$ is observed to be effective. Similarly, degeneracy effects are seen to be operational at high fields and in densely populated regions. Non-uniform electric field profiles and transient evolution of the electron density along the device are analysed. Finally, at very high fields II is shown to introduce a substantial energy loss mechanism for the energetic carriers that have just traversed the unintentionally doped n-region.

\section{Acknowledgments}

This work is supported by The Scientific and Technical Research Council of Turkey (TÜBITAK).

\section{References}

I BULUTAY, C.: 'Electson initiated impact ionization in AJGaN alloys', Semicond. Sci. Technol.. 2002, 17, (10), pp. L59-L.62

2 FAWCETT W, BOARDMAN, A.D., and SWAIN, S. 'Monte Carlo determination of electron transport properties in gallium arsenide'. determination of electron transport properties in
J. Phys. Chem. Solids, 1970, 31, pp. 1963-1990

3 BULUTAY, C., RIDLEY, B.K., and ZAKHLENIUK, N.A.: 'Full-band polar optical phonon scattering analysis and negative differential conductivity in wurtzite GaN', Phys. Rev: B, 2000, 62, (23), pp. 15754-15763

4 BULUTAY, C. RIDLEY, B.K and ZAKHLENIUK, N.A.: 'Highenergy electron relaxation and full band electron dynamics in aluminium nitride', Physica B. 2002, 314, (1 -4), pp. 63-67

5 FARAHMAND, M., GARETTO, C., BELLOTTI, E., BRENNAN, K.F GOANO, M.. GHILLINO, E., GHIONE, G., ALBRECHT, J.D., and RUDEN, P.P.: 'Monte Carlo simulation of electron transport in the III-nitride wurtzite phase matcrials system: binaries and ternaries', IEEE Trans. Electron Devices, 2001, 48, (3), pp. 535-542

6 FISCHETTI, M.V., and LAUX, S.E.: 'Band structure, deformation potentials and carrier mobility in strained $\mathrm{Si}$, Ge and SiGe alloys', J. Appl. Phys, 1996, 80, (4), pp. 2234-2252

7 TOMIZAWA, K., AWANO, Y., HASHIZUME, N., and KAWASHIMA, M.: 'Monte Carlo simulation of submicron GaAs $n^{+}-i(n)-n^{+}$diode', Proc. IEE, 1982, 129, pp. 13!-136

8 LUGLI, P. and FERRY, D.K.: 'Degeneracy in the ensemble Monte Carlo method for high-field transport', IEEE Truns. Electron Devices, 1985, 32, (11), pp. 2431-2437 